\title{
Study of a Biparametric Family of Iterative Methods
}

\author{
B. Campos, ${ }^{1}$ A. Cordero, ${ }^{2}$ Á. A. Magreñán, ${ }^{3}$ J. R. Torregrosa, ${ }^{2}$ and P. Vindel $^{1}$ \\ ${ }^{1}$ Instituto de Matemáticas y Aplicaciones de Castellón, Universitat Jaume I, 12071 Castellón, Spain \\ ${ }^{2}$ Instituto de Matemática Multidisciplinar, Universitat Politécnica de Valencia, 46022 Valencia, Spain \\ ${ }^{3}$ Universidad Internacional de la Rioja, 26002 Logroño, Spain
}

Correspondence should be addressed to P. Vindel; vindel@uji.es

Received 4 June 2014; Revised 25 July 2014; Accepted 27 July 2014; Published 24 December 2014

Academic Editor: Jisheng Kou

Copyright (C) 2014 B. Campos et al. This is an open access article distributed under the Creative Commons Attribution License, which permits unrestricted use, distribution, and reproduction in any medium, provided the original work is properly cited.

\begin{abstract}
The dynamics of a biparametric family for solving nonlinear equations is studied on quadratic polynomials. This biparametric family includes the $c$-iterative methods and the well-known Chebyshev-Halley family. We find the analytical expressions for the fixed and critical points by solving 6-degree polynomials. We use the free critical points to get the parameter planes and, by observing them, we specify some values of $(\alpha, c)$ with clear stable and unstable behaviors.
\end{abstract}

\section{Introduction}

Iterative methods are needed for solving the most of the nonlinear equations $f(x)=0$, because they are difficult or impossible to solve exactly by means of analytical methods. When they are applied on polynomials, they give rise to rational functions whose behavior is not well known except in small areas. A way to extend these regions is by studying the dynamical behavior of the rational functions associated to the iterative methods. In some previous papers, we focus on this option and we start with the dynamical study of ChebyshevHalley family ([1-3]), the King's family [4], and the $c$-family [5].

The $(\alpha, c)$-family is a biparametric family of iterative methods that includes the Chebyshev-Halley $([6,7])$ and $c$ families when one of the parameters is fixed.

In [8] the authors establish the conjugacy classes for $(\alpha, c)$-family and they find explicit formulations for fixed and critical points of the Chebyshev, Halley, and super Halley methods and also for the $c$-family, applied on an arbitrary polynomial.

In this paper, we consider the $(\alpha, c)$-family and we calculate the fixed and critical points of this family applied on quadratic polynomials. By solving 6-degree polynomials we obtain the analytical expressions for the fixed and critical points of this family in terms of both parameters.
From these analytical expressions we make a study of the points in the $(\alpha, c)$-plane for $\alpha$ and $c$ real. The analysis of the critical points helps us to obtain the parameter planes. From these parameter planes we specify some values of $(\alpha, c)$ with clear stable and unstable behavior and show the dynamical planes of the corresponding iterative methods.

The $(\alpha, c)$-family (see [9]) is a two-parametric third-order class of iterative methods defined by

$$
z_{n+1}=z_{n}-\left(1+\frac{1}{2} \frac{L_{f}\left(z_{n}\right)}{1-\alpha L_{f}\left(z_{n}\right)}+c L_{f}\left(z_{n}\right)^{2}\right) \frac{f\left(z_{n}\right)}{f^{\prime}\left(z_{n}\right)},
$$

where

$$
L_{f}(z)=\frac{f(z) f^{\prime \prime}(z)}{f^{\prime}(z)^{2}}
$$

and parameters $\alpha$ and $c$ are complex. This family includes Chebyshev-Halley family for $c=0$ and $c$-family when $\alpha=0$. As it is seen throughout this paper, the dynamical behavior of this family is much more complicated than previously studied because it includes two parameters $\alpha$ and $c$.

For beginning the study of the dynamics of this family, we apply the operator associated to (1) on quadratic polynomials. Taking into account that this family satisfies the scaling 
theorem (see [9]), the rational operators obtained when applying the method on every quadratic polynomial are conjugated to the one obtained on $p(z)=z^{2}+a$. For this polynomial, the operator $M_{p}(z, \alpha, c, a)$ associated to (1) is a rational function depending on three complex parameters, $a$, $\alpha$, and $c$ :

$$
\begin{aligned}
& M(z, \alpha, c, a) \\
& =-\left(\alpha c a^{4}+2(-1+2 \alpha) c a^{3} z^{2}\right. \\
& \quad+2(-1+2 \alpha-3 c+3 \alpha c) a^{2} z^{4} \\
& \left.\quad+2(-6-3 c+2 \alpha c) a z^{6}+(6-4 \alpha-2 c+\alpha c) z^{8}\right) \\
& \times\left(8 z^{5}\left(\alpha a-2 z^{2}+\alpha z^{2}\right)\right)^{-1} .
\end{aligned}
$$

Rational functions $R$ and $g \circ R \circ g^{-1}$ are conjugated, $g$ being a Möebius map. Considering $g(z)=(z+i \sqrt{a}) /(z-i \sqrt{a})$ the parameter $a$ can be obviated and the operator $M(z, \alpha, c, a)$ is conjugated to

$$
\begin{aligned}
& O_{p}(z, \alpha, c) \\
& \quad=z^{3} \frac{(1+z)^{4}(-2+2 \alpha-z)+4 c(1+z(2-2 \alpha+z))}{(1+z)^{4}(2 \alpha z-1-2 z)+4 c z^{3}(1+z)^{2}-8 \alpha c z^{4}} .
\end{aligned}
$$

By using this Möebius map, the roots of $p(z)$ become 0 and $\infty$; meanwhile $g(\infty)=1$.

Iterative methods are used for finding the roots of a nonlinear equation and, from a dynamical point of view, these roots are included in the set of the fixed points of the operator associated with the method. So, from this point of view, our greatest interest is to find the fixed points of operator (4) and to study their stability.

The natural space to study the dynamics of a rational map $R$ is the Riemann sphere $\widehat{\mathbb{C}}, R: \widehat{\mathbb{C}} \rightarrow \widehat{\mathbb{C}}$ (see $[10,11]$, e.g.). The dynamics of this rational map induces a subdivision of the complex sphere into two sets, namely, the Fatou set $\mathscr{F}(R)$ and the Julia set $\mathscr{J}(R)$ of $R$. The Fatou set of a nonconstant rational function $R$ is the maximal open subset of $\widehat{\mathbb{C}}$ on which the family of iterates $\left\{R^{n}\right\}$ is equicontinuous (roughly speaking, they preserve the proximity of points) and the Julia set is its complement in $\widehat{\mathbb{C}}$. From this definition, we know that $\mathscr{F}(R)$ is open and $\mathscr{J}(R)$ is compact. Moreover, if $S=g \circ R \circ g^{-1}$, where $g$ is a Möebius map, then $F(S)=g(F(R))$ and $J(S)=g(J(R))$ ([11, Theorem 3.1.4]).

Let us recall some dynamical concepts of complex dynamics that we use in this paper (see [10]). Given a rational function $R: \widehat{\mathbb{C}} \rightarrow \widehat{\mathbb{C}}$, the orbit of a point $z_{0} \in \widehat{\mathbb{C}}$ is defined as

$$
\left\{z_{0}, R\left(z_{0}\right), R^{2}\left(z_{0}\right), \ldots, R^{n}\left(z_{0}\right), \ldots\right\} .
$$

We analyze the phase plane of the map $R$ by classifying the starting points from the asymptotic behavior of their orbits. A point $z_{0} \in \widehat{\mathbb{C}}$ is called a fixed point if $R\left(z_{0}\right)=z_{0}$. A periodic point $z_{0}$ of period $p>1$ is a point such that $R^{p}\left(z_{0}\right)=z_{0}$ and $R^{k}\left(z_{0}\right) \neq z_{0}$, for $k<p$. A preperiodic point is a point $z_{0}$ that is not periodic, but there exists a $k>0$ such that $R^{k}\left(z_{0}\right)$ is periodic. A point $z_{0}$ is a critical point of the rational map $R$ if $R$ fails to be injective in any neighborhood of $z_{0}$. Moreover, a fixed point $z_{0}$ is called attractor if $\left|R^{\prime}\left(z_{0}\right)\right|<1$, superattractor if $\left|R^{\prime}\left(z_{0}\right)\right|=0$, repulsor if $\left|R^{\prime}\left(z_{0}\right)\right|>1$, and parabolic if $\left|R^{\prime}\left(z_{0}\right)\right|=1$. The fixed points different from the roots of the polynomial $p(z)$ are called strange fixed points.

The basin of attraction of an attractor $\alpha$ is defined as

$$
\mathscr{A}(\alpha)=\left\{z_{0} \in \widehat{\mathbb{C}}: R^{n}\left(z_{0}\right) \longrightarrow \alpha, n \longrightarrow \infty\right\} .
$$

The Fatou set of the rational function $R$ is the set of points $z \in \widehat{\mathbb{C}}$ whose orbits tend to an attractor (fixed point, periodic orbit, or infinity). Since its complement in $\widehat{\mathbb{C}}$ is the Julia set the basin of attraction of any fixed point belongs to the Fatou set and the boundaries of these basins of attraction belong to the Julia set.

The rest of the paper is organized as follows. In Section 2 we make a preliminary study of the fixed points, obtaining their analytic expressions and presenting the regions of the $(\alpha, c)$-plane where they are complex or real. Section 3 is devoted to the analysis of the critical points, which will be useful in Section 5 to obtain the parameter planes. In Section 4 we check that the obtained results are consistent with the known ones of Chebyshev-Halley class and $c$-family. Section 6 shows some dynamical planes where interesting qualitative behaviors appear. Finally, in Section 7, some conclusions are stated.

\section{Study of the Fixed Points}

The fixed points of the operator (4) are obtained by solving the equation $O_{p}(z, \alpha, c)=z$. Evaluating $O_{p}(z, \alpha, c)-z$ we have to solve the equation

$$
\begin{aligned}
& -z(z-1) \frac{P(z, \alpha, c)}{(1+z)^{4}(2 \alpha z-1-2 z)+4 c z^{3}(1+z)^{2}-8 \alpha c z^{4}} \\
& \quad=0,
\end{aligned}
$$

where $P(z, a, c)$ is the symmetric 6-degree polynomial:

$$
\begin{aligned}
P(z, a, c)= & 1+(7-2 \alpha) z+(19-8 \alpha+4 c) z^{2} \\
& +(26-12 \alpha+8 c-8 \alpha c) z^{3}+(19-8 \alpha+4 c) z^{4} \\
& +(7-2 \alpha) z^{5}+z^{6} .
\end{aligned}
$$

The solutions $z=0$ and $z=\infty$ of (7) are associated to the roots of $p(z)$. We observe that $z=1$ is a strange fixed point for every value of the parameters. The other fixed points come from the roots of $P(z, a, c)$. These roots must include the strange fixed points of ChebyshevHalley family for $c=0$ and the respective ones of $c$ family for $\alpha=0$, as we will see in Section 4 . In the following result we present the explicit expressions of the strange fixed points. An exhaustive proof can be found in [12]. 
Theorem 1. The fixed points of the operator (4), associated to the biparametric family of iterative methods (1) on quadratic polynomials, are:

(i) $z=0$ and $z=\infty$, corresponding to the roots of the polynomial;

(ii) seven strange fixed points: $z=1$ and the following six points:

$$
\begin{aligned}
& z_{1}(\alpha, c)=\frac{x_{1}(\alpha, c)+\sqrt{x_{1}(\alpha, c)^{2}-4}}{2}, \\
& z_{2}(\alpha, c)=\frac{x_{1}(\alpha, c)-\sqrt{x_{1}(\alpha, c)^{2}-4}}{2}, \\
& z_{3}(\alpha, c)=\frac{x_{2}(\alpha, c)+\sqrt{x_{2}(\alpha, c)^{2}-4}}{2}, \\
& z_{4}(\alpha, c)=\frac{x_{2}(\alpha, c)-\sqrt{x_{2}(\alpha, c)^{2}-4}}{2}, \\
& z_{5}(\alpha, c)=\frac{x_{3}(\alpha, c)+\sqrt{x_{3}(\alpha, c)^{2}-4}}{2} \\
& z_{6}(\alpha, c)=\frac{x_{3}(\alpha, c)-\sqrt{x_{3}(\alpha, c)^{2}-4}}{2}
\end{aligned}
$$

where

$$
\begin{aligned}
x_{1}(\alpha, c)= & \frac{1}{3}(2 \alpha-7)+\frac{1}{3}(\sqrt[3]{f(\alpha, c)}+\sqrt[3]{g(\alpha, c)}), \\
x_{2}(\alpha, c)= & \frac{1}{3}(2 \alpha-7)-\frac{1}{6}(\sqrt[3]{f(\alpha, c)}+\sqrt[3]{g(\alpha, c)}) \\
& -i \frac{\sqrt{3}}{6}(\sqrt[3]{f(\alpha, c)}-\sqrt[3]{g(\alpha, c)}), \\
x_{3}(\alpha, c)= & \frac{1}{3}(2 \alpha-7)-\frac{1}{6}(\sqrt[3]{f(\alpha, c)}+\sqrt[3]{g(\alpha, c)}) \\
& +i \frac{\sqrt{3}}{6}(\sqrt[3]{f(\alpha, c)}-\sqrt[3]{g(\alpha, c)}),
\end{aligned}
$$

$f(\alpha, c)$

$$
\begin{aligned}
= & (-1+2 \alpha)^{3}+18(1+4 \alpha) c \\
& +6 \sqrt{3} \sqrt{c\left(2 \alpha(-1+2 \alpha)^{3}+c\left(-1+40 \alpha+32 \alpha^{2}+16 c\right)\right)},
\end{aligned}
$$

$g(\alpha, c)$

$$
\begin{aligned}
= & (-1+2 \alpha)^{3}+18(1+4 \alpha) c \\
& -6 \sqrt{3} \sqrt{c\left(2 \alpha(-1+2 \alpha)^{3}+c\left(-1+40 \alpha+32 \alpha^{2}+16 c\right)\right)} .
\end{aligned}
$$

Proof. The fixed points satisfy $O_{p}(z, \alpha, c)=z$. As we have seen, the numerator of $O_{p}(z, \alpha, c)-z$ can be factorized as

$$
-z(z-1) P(z, a, c),
$$

where $P(z, a, c)$ is the polynomial given in (8).
The fixed points $z=0$ and $z=1$ are trivially obtained. Now, we look for the roots of the 6-degree polynomial (8). The change of variable $x=z+1 / z$ transforms polynomial (8) into the cubic one:

$$
x^{3}+(7-2 \alpha) x^{2}+(16-8 \alpha+4 c) x+12-8 \alpha+8 c-8 \alpha c
$$

whose roots are

$$
\begin{aligned}
x_{1}(\alpha, c)= & \frac{1}{3}(-7+2 \alpha)+\frac{1}{3}(\sqrt[3]{f(\alpha, c)}+\sqrt[3]{g(\alpha, c)}), \\
x_{2}(\alpha, c)= & \frac{1}{3}(-7+2 \alpha)-\frac{1}{6}(\sqrt[3]{f(\alpha, c)}+\sqrt[3]{g(\alpha, c)}) \\
& -i \frac{\sqrt{3}}{6}(\sqrt[3]{f(\alpha, c)}-\sqrt[3]{g(\alpha, c)}) \\
x_{3}(\alpha, c)= & \frac{1}{3}(-7+2 \alpha)-\frac{1}{6}(\sqrt[3]{f(\alpha, c)}+\sqrt[3]{g(\alpha, c)}) \\
& +i \frac{\sqrt{3}}{6}(\sqrt[3]{f(\alpha, c)}-\sqrt[3]{g(\alpha, c)})
\end{aligned}
$$

where

$$
\begin{gathered}
f(\alpha, c)=(-1+2 \alpha)^{3}+18(1+4 \alpha) c-6 \sqrt{3} \sqrt{\Delta}, \\
g(\alpha, c)=(-1+2 \alpha)^{3}+18(1+4 \alpha) c+6 \sqrt{3} \sqrt{\Delta}, \\
\Delta=c\left(2 \alpha(-1+2 \alpha)^{3}+c\left(-1+40 \alpha+32 \alpha^{2}+16 c\right)\right) .
\end{gathered}
$$

Undoing the change of variable, the six roots of the polynomial $P(z, \alpha, c)$ are obtained by solving the quadratic equation:

$$
z^{2}-z x+1=0
$$

whose solutions are

$$
z_{ \pm}=\frac{x \pm \sqrt{x^{2}-4}}{2} .
$$

That is, the six roots of (8) are obtained by substituting in (16) the values of $x$ given in (13).

From Cardano's method we know that, $\alpha$ and $c$ being real:

(i) if $\Delta>0$ there is a unique real solution and a pair of complex conjugated roots;

(ii) if $\Delta=0$ there are two different real solutions, one of them double; and there is one real solution with multiplicity three $(x=(-7+2 \alpha) / 3)$ when the following system of equations is fulfilled:

$$
\begin{gathered}
\Delta=0, \\
(-1+2 \alpha)^{3}+18(1+4 \alpha) c=0 ;
\end{gathered}
$$

the only values of the parameters that satisfy this system are

$$
\left(\alpha=\frac{1}{2}, c=0\right), \quad\left(a=\frac{-1}{16}, c=\frac{27}{256}\right) ;
$$

(iii) if $\Delta<0$ there are three different real solutions. 


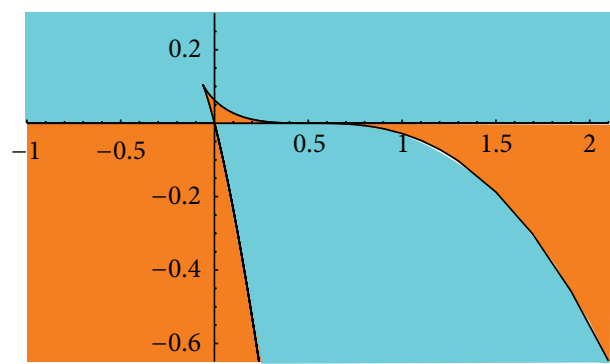

Figure 1: The curves $C_{-}$and $C_{+}$.

So, $(\alpha, c)$-plane is divided into two regions, as we see in Figure 1. In this figure, the orange region corresponds to $\Delta<0$ and the blue region corresponds to $\Delta>0$. They are delimited by the axis $c=0$ and the curve $\Delta=0$. This curve is formed by the graphics of the two functions:

$$
C_{ \pm}=\frac{1-40 a-32 a^{2} \pm \sqrt{(16 a+1)^{3}}}{32} .
$$

Moreover, from (16), if a point $x_{i}(\alpha, c), i=1,2,3$, is real, it must be satisfied $x_{i}(\alpha, c) \geq 2$ or $x_{i}(\alpha, c) \leq-2$ in order to be real the corresponding fixed points $z_{i}(\alpha, c)$. So, we look for the separating curves $x_{1}(\alpha, c)=2$ and $x_{1}(\alpha, c)=-2$ :

$$
\begin{aligned}
& x_{1}(\alpha, c) \\
& \quad=2 \Longrightarrow \frac{1}{3}(-7+2 \alpha)+\frac{1}{3}(\sqrt[3]{f(\alpha, c)}+\sqrt[3]{g(\alpha, c)})=2 .
\end{aligned}
$$

Raising to the cube both sides of the equality and taking into account that

$$
\begin{aligned}
&(\sqrt[3]{f(\alpha, c)}+\sqrt[3]{g(\alpha, c)})^{3} \\
&= f(\alpha, c)+g(\alpha, c) \\
&+3 \sqrt[3]{f(\alpha, c) g(\alpha, c)}(\sqrt[3]{f(\alpha, c)}+\sqrt[3]{g(\alpha, c)}) \\
&= f(\alpha, c)+g(\alpha, c) \\
&+3\left(1-4 \alpha+4 \alpha^{2}-12 c\right)(\sqrt[3]{f(\alpha, c)}+\sqrt[3]{g(\alpha, c)}),
\end{aligned}
$$

we obtain

$$
216(\alpha(4+c)-2(5+c))=0 \Longrightarrow c=\frac{2(-5+2 \alpha)}{2-\alpha} .
$$

Similarly,

$$
x_{1}(\alpha, c)=-2 \Longrightarrow 216 \alpha c=0 \Longrightarrow c=0 \quad \text { or } \quad \alpha=0
$$

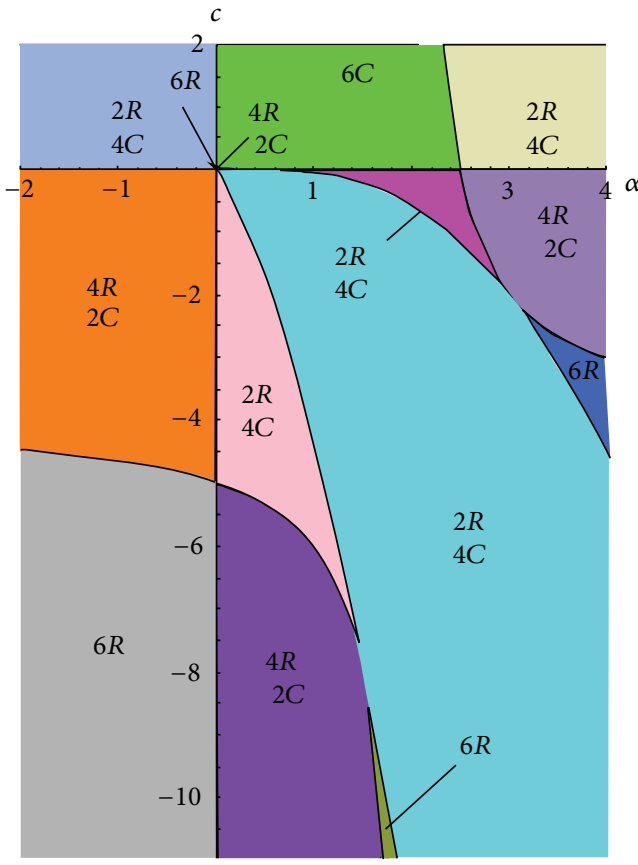

FIGURE 2: Bifurcation curves of fixed points.

It can be checked that the same separating curves appear when we consider $x_{2}(\alpha, c)= \pm 2$ and $x_{3}(\alpha, c)= \pm 2$. In Figure 2, we observe the different regions separated by these curves and the number of real and complex fixed points in each region. Let us note that the fixed points change their nature, from complex to real or vice versa, when they cross these curves. For example, for $\alpha=-1$, there are six real roots for negative values of $c$ below the curve $c=$ $2(-5+2 \alpha) /(2-\alpha)$; two of them change to complex when they cross this curve and other two roots change from real to complex when crossing the axis $c=0$ (see Figure 3). An exhaustive study of these bifurcations has been made in [12].

\section{Study of Critical Points}

Critical points satisfy $O_{p}^{\prime}(z, \alpha, c)=0$, where the prime means the derivative of $O_{p}(z, \alpha, c)$ with respect to $z$. The expression of this derivative is

$$
\begin{aligned}
& O_{p}^{\prime}(z, \alpha, c) \\
& \quad=\frac{-2 z^{2}(1+z)^{4} Q(z, \alpha, c)}{\left((1+z)^{4}(2 \alpha z-1-2 z)+4 c z^{3}(1+z)^{2}-8 \alpha c z^{4}\right)^{2}},
\end{aligned}
$$

where

$$
Q(z, a, c)=b_{0}+b_{1} z+b_{2} z^{2}+b_{3} z^{3}+b_{2} z^{4}+b_{1} z^{5}+b_{0} z^{6}
$$




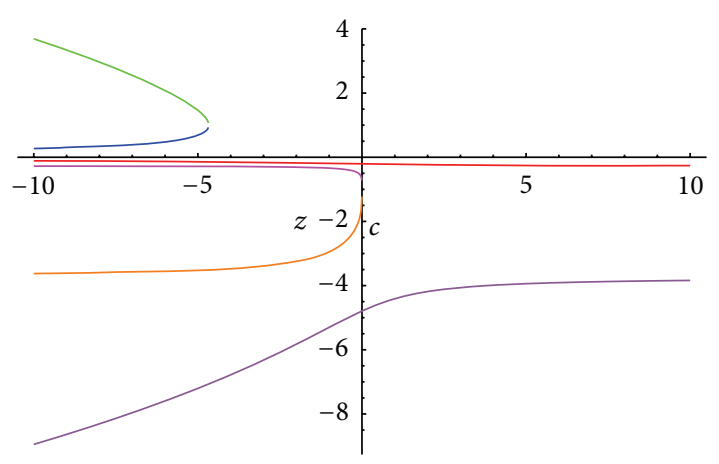

Figure 3: Bifurcation diagram of fixed points for $\alpha=-1$.

$$
\begin{aligned}
& b_{0}=-3+3 \alpha+6 c \\
& b_{1}=-18+20 \alpha-4 \alpha^{2}+16 c-24 \alpha c, \\
& b_{2}=-45+53 \alpha-16 \alpha^{2}+10 c-16 \alpha c+24 \alpha^{2} c, \\
& b_{3}=-60+72 \alpha-24 \alpha^{2}+16 \alpha c-32 \alpha^{2} c .
\end{aligned}
$$

The solutions $z=0$ and $z=\infty$ are associated to the roots of $p(z)$, which are always super attractors because methods of family (1) have order of convergence three. The expressions of the other critical points come from the roots of the 6-degree symmetric polynomial $Q(z, a, c)$ given in the following result.

Theorem 2. The critical points of the operator $O_{p}(z, \alpha, c)$, associated to the biparametric family of iterative methods (1) on quadratic polynomials, are

(i) $z=0$ and $z=\infty$, which are associated to the nonstrange fixed points;

(ii) seven free critical points: $z=-1$ and

$$
\begin{aligned}
& z_{1}(\alpha, c)=\frac{x_{1}(\alpha, c)+\sqrt{x_{1}(\alpha, c)^{2}-4}}{2}, \\
& z_{2}(\alpha, c)=\frac{x_{1}(\alpha, c)-\sqrt{x_{1}(\alpha, c)^{2}-4}}{2}, \\
& z_{3}(\alpha, c)=\frac{x_{2}(\alpha, c)+\sqrt{x_{2}(\alpha, c)^{2}-4}}{2}, \\
& z_{4}(\alpha, c)=\frac{x_{2}(\alpha, c)-\sqrt{x_{2}(\alpha, c)^{2}-4}}{2}, \\
& z_{5}(\alpha, c)=\frac{x_{3}(\alpha, c)+\sqrt{x_{3}(\alpha, c)^{2}-4}}{2} \\
& z_{6}(\alpha, c)=\frac{x_{3}(\alpha, c)-\sqrt{x_{3}(\alpha, c)^{2}-4}}{2},
\end{aligned}
$$

where

$$
\begin{aligned}
& x_{1}(\alpha, c)=-\frac{b_{1}}{3 b_{0}}+s_{1}+s_{2}, \\
& x_{2}(\alpha, c)=-\frac{b_{1}}{3 b_{0}}-\frac{1}{2}\left(s_{1}+s_{2}\right)+i \frac{\sqrt{3}}{2}\left(s_{1}-s_{2}\right),
\end{aligned}
$$

$$
\begin{aligned}
x_{3}(\alpha, c) & =-\frac{b_{1}}{3 b_{0}}-\frac{1}{2}\left(s_{1}+s_{2}\right)-i \frac{\sqrt{3}}{2}\left(s_{1}-s_{2}\right), \\
s_{1} & =\sqrt[3]{\frac{-q+\sqrt{D}}{2}}, \quad p=-3+\frac{b_{2}}{b_{0}}-\frac{b_{1}^{2}}{3 b_{0}^{2}}, \\
s_{2} & =\sqrt[3]{\frac{-q-\sqrt{D}}{2}}, \quad q=\frac{b_{3}-b_{1}}{b_{0}}-\frac{b_{1} b_{2}}{3 b_{0}^{2}}+\frac{2 b_{1}^{3}}{27 b_{0}^{3}}, \\
D & =\frac{4}{27} p^{3}+q^{2} .
\end{aligned}
$$

Proof. The critical points $z=0,-1, \infty$ are obtained immediately. So, we search the roots of the polynomial $Q(z, \alpha, c)$ in operator (24). For $b_{0} \neq 0$, the solutions of $Q(z, a, c)=0$ are the same of

$$
1+a_{1} z+a_{2} z^{2}+a_{3} z^{3}+a_{2} z^{4}+a_{1} z^{5}+z^{6}=0,
$$

where $a_{i}=b_{i} / b_{0}, i=1,2,3$, and $\alpha \neq 1-2 c$.

As $z=0$ is not a root of the polynomial (25), we can divide the previous equation by $z^{3}$ and use the change of variable $x=z+1 / z$, which leads to the cubic polynomial equation:

$$
x^{3}+\bar{a} x^{2}+\bar{b} x+\bar{c}=0
$$

where

$$
\bar{a}=a_{1}=\frac{b_{1}}{b_{0}}, \quad \bar{b}=\left(-3+\frac{b_{2}}{b_{0}}\right), \quad \bar{c}=\frac{b_{3}-2 b_{1}}{b_{0}} .
$$

The quadratic term is eliminated by means of the change

$$
x=y-\frac{\bar{a}}{3},
$$

and the final polynomial equation is

$$
y^{3}+p y+q=0
$$

where

$$
\begin{aligned}
& p=\bar{b}-\frac{\bar{a}^{2}}{3}=-3+\frac{b_{2}}{b_{0}}-\frac{b_{1}^{2}}{3 b_{0}^{2}}, \\
& q=\frac{2 \bar{a}^{3}}{27}-\frac{\bar{a} \bar{b}}{3}+\bar{c}=\frac{b_{3}-b_{1}}{b_{0}}-\frac{b_{1} b_{2}}{3 b_{0}^{2}}+\frac{2 b_{1}^{3}}{27 b_{0}^{3}} .
\end{aligned}
$$

By using the change $y=s_{1}+s_{2}, y^{3}=s_{1}^{3}+s_{2}^{3}+3 s_{1} s_{2} y$ is obtained. By identifying the coefficients of this equation with the coefficients of (33) we obtain

$$
\begin{gathered}
s_{1}^{3}+s_{2}^{3}=-q, \\
s_{1}^{3} s_{2}^{3}=-\frac{p^{3}}{27} .
\end{gathered}
$$

So, $s_{1}^{3}$ and $s_{2}^{3}$ are solutions of the quadratic equation $W^{2}+q W-$ $p^{3} / 27=0$; that is,

$$
s_{1}^{3}, s_{2}^{3}=\frac{-q \pm \sqrt{q^{2}+4 p^{3} / 27}}{2} .
$$


Therefore, the three solutions of (33) are

$$
\begin{aligned}
& y_{1}=s_{1}+s_{2}, \\
& y_{2}=-\frac{1}{2}\left(s_{1}+s_{2}\right)+i \frac{\sqrt{3}}{2}\left(s_{1}-s_{2}\right), \\
& y_{3}=-\frac{1}{2}\left(s_{1}+s_{2}\right)-i \frac{\sqrt{3}}{2}\left(s_{1}-s_{2}\right),
\end{aligned}
$$

where

$$
\begin{gathered}
s_{1}=\sqrt[3]{\frac{-q+\sqrt{D}}{2}}, \quad s_{2}=\sqrt[3]{\frac{-q-\sqrt{D}}{2}}, \\
D=\frac{4}{27} p^{3}+q^{2} .
\end{gathered}
$$
from

Undoing all the changes, the six roots of (25) are obtained

$$
z=\frac{x \pm \sqrt{x^{2}-4}}{2}
$$

for the different values of variable $x$.

The number of real and complex roots of (33) depends on the sign of $D$. If $D>0$, there are one real and two complex roots, meanwhile if $D \leq 0$, the three roots are real.

As

$$
\begin{gathered}
D=\left(-64 \alpha^{3} c\left(-10 \alpha^{2}(-1+2 \alpha)^{3}\right.\right. \\
+\alpha\left(-3575+5360 \alpha-2696 \alpha^{2}\right. \\
\left.+384 \alpha^{3}+36 \alpha^{4}\right) c \\
\left.\left.-8(-2+\alpha)(-5+3 \alpha)^{3} c^{2}\right)\right) \\
\times\left(2187(-1+\alpha+2 c)^{4}\right)^{-1}
\end{gathered}
$$

the curve $D=0$ implies $\alpha=0, c=0$, or

$$
\begin{aligned}
& -10 \alpha^{2}(-1+2 \alpha)^{3} \\
& +\alpha\left(-3575+5360 \alpha-2696 \alpha^{2}+384 \alpha^{3}+36 \alpha^{4}\right) c \\
& -8(-2+\alpha)(-5+3 \alpha)^{3} c^{2}=0 .
\end{aligned}
$$

This last equation defines two functions:

$$
\begin{aligned}
C^{ \pm}=( & -\alpha\left(\left(-3575+5360 \alpha-2696 \alpha^{2}+384 \alpha^{3}+36 \alpha^{4}\right)\right. \\
& \left.\left. \pm \alpha \sqrt{3\left(5-6 \alpha+2 \alpha^{2}\right)\left(95-58 \alpha+6 \alpha^{2}\right)^{3}}\right)\right) \\
& \times\left(16(-2+\alpha)(-5+3 \alpha)^{3}\right)^{-1},
\end{aligned}
$$

whose domain is $\{\alpha: \alpha \leq(1 / 6)(29-\sqrt{271}) \cup \alpha \geq(1 / 6)(29+$ $\sqrt{271})\}$. We can check that $\alpha=2$ is an asymptote of $c^{+}$and

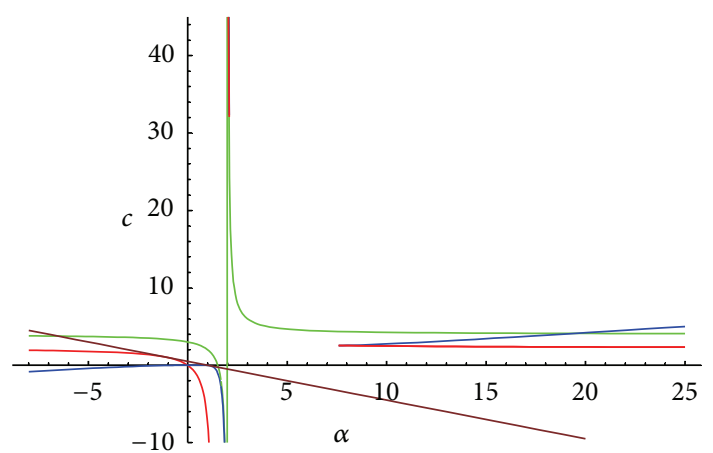

FIgURE 4: Bifurcation curves of critical points.

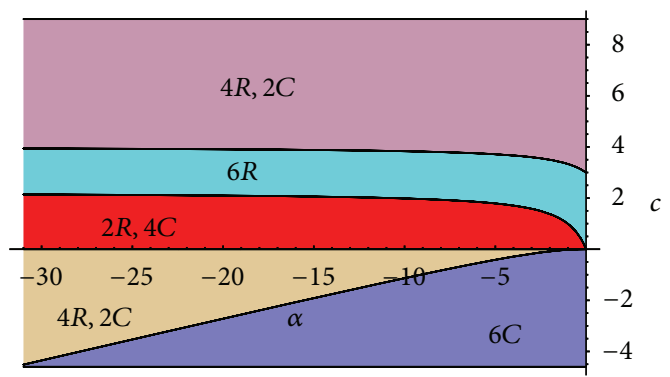

Figure 5: Critical points in the $(\alpha, c)$-plane for $\alpha<0$.

$\alpha=5 / 3$ is an asymptote of $C^{-}$; moreover, $C^{-}$has the horizontal asymptote $c=20 / 9$ and $C^{+}$has not any horizontal asymptote.

Taking into account the change (39), the number of real and complex roots also depends on whether $x \geq 2$ or $x \leq-2$. Taking $x=2$, we get

$$
s_{1}+s_{2}=\frac{6+a_{1}}{3} \Longrightarrow\left(s_{1}+s_{2}\right)^{3}=\left(\frac{6+a_{1}}{3}\right)^{3} .
$$

As $s_{1} s_{2}=-p / 3$ and $s_{1}^{3}+s_{2}^{3}=-q$, we obtain the equation

$$
\frac{16(\alpha-2)(6+\alpha(-4+c)-2 c)}{3(-1+\alpha+2 c)}=0 .
$$

So, if $-1+\alpha+2 c \neq 0$, we obtain the bifurcation curves $\alpha=2$ and $c=2(2 \alpha-3) /(\alpha-2), \alpha \neq 2$.

Taking $x=-2$, then

$$
-\frac{80 \alpha^{2} c}{3(-1+\alpha+2 c)}=0 .
$$

So, if $-1+\alpha+2 c \neq 0$, we obtain the bifurcation curves $\alpha=0$ and $c=0$. All these bifurcation curves can be seen in Figure 4.

These curves separate the $(\alpha, c)$-plane into different regions with different number of real and complex critical points. These regions are shown in Figures 5, 6, and 7. 


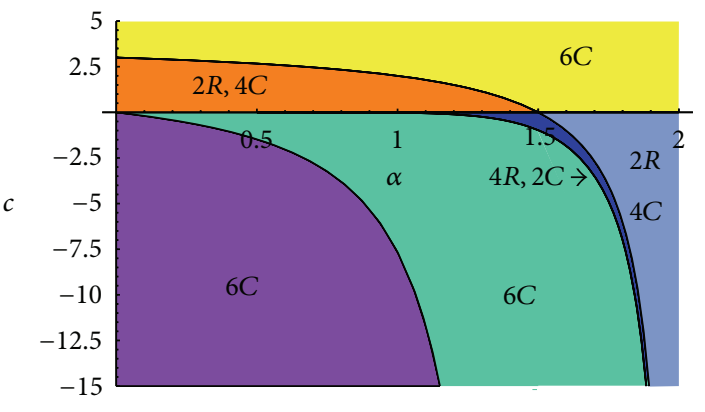

(a) $0<\alpha<2$

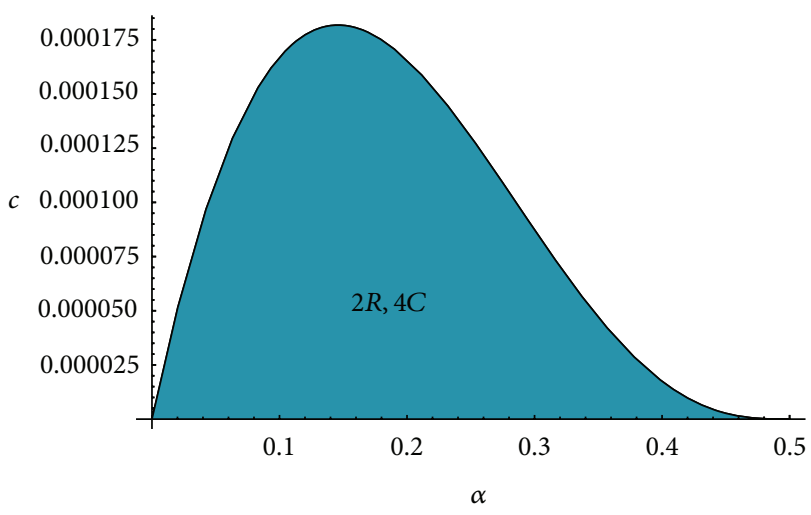

(b) Detail of the zone for $0<\alpha<0.5$

Figure 6: Critical points in the $(\alpha, c)$-plane for $0<\alpha<2$.

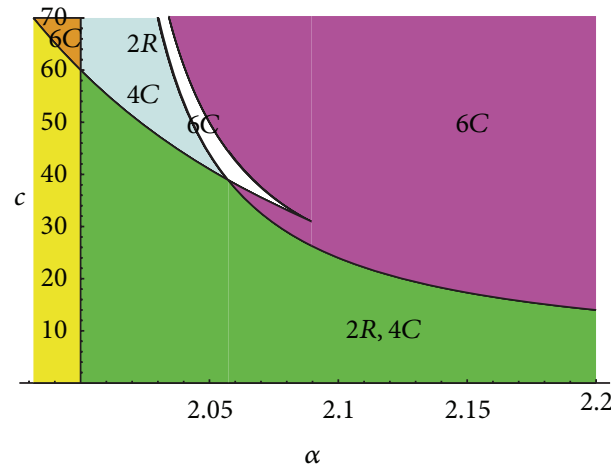

(a) $1.9<\alpha<2.2$

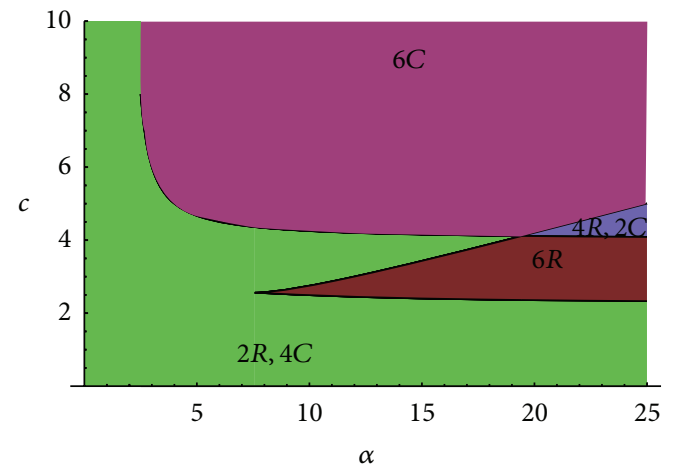

(b) $\alpha>2.2$

FIgURE 7: Critical points in the $(\alpha, c)$-plane for $1.9<\alpha$ and $c>0$.

Let us remark that there are only three free critical points because they satisfy the relationships:

$$
z_{1} z_{2}=1, \quad z_{3} z_{4}=1, \quad z_{5} z_{6}=1,
$$

for every values $(\alpha, c)$.

If $-1+\alpha+2 c=0$, then polynomial (25) becomes a product of $z$ and a symmetric 4-degree polynomial. Following the same steps as before, on the line $\alpha=1-2 c$ and $\alpha \neq \pm \sqrt{5} / 2$, we have for $\alpha<(1 / 2)(-5-\sqrt{65})$ two real and two complex roots, for $(1 / 2)(-5-\sqrt{65})<\alpha<0$ four real roots, for $0<\alpha<1$ four complex roots, for $1<\alpha<(1 / 2)(-5+\sqrt{65})$ two real and two complex roots, for $(1 / 2)(-5+\sqrt{65})<\alpha<2$ four complex roots, and for $\alpha>2$ two real and two complex roots. Finally, for $\alpha= \pm \sqrt{5} / 2$, polynomial (25) yields a symmetric 2-degree polynomial. For $\alpha=-\sqrt{5} / 2$ both roots are complex and for $\alpha=\sqrt{5} / 2$ both roots are real.

\section{Chebyshev-Halley Class and $c$-Family of Iterative Methods}

We know that Chebyshev-Halley class and $c$-family of iterative methods are included in the biparametric family (1). In this section, we prove that the 6-degree polynomial (8) can be factorized as a product of a polynomial of degree two and a polynomial of degree four, whose roots correspond to the fixed points of Chebyshev family for $c=0$ and $c$-family for $\alpha=0$, respectively.

So,

$$
P(z, \alpha, c)=h_{1}(z, \alpha, c) h_{2}(z, \alpha, c),
$$

where

$$
\begin{aligned}
& h_{1}(z, \alpha, c)=1+d z+z^{2} \\
& h_{2}(z, \alpha, c)=1+d_{1} z+d_{2} z^{2}+d_{3} z^{3}+z^{4}
\end{aligned}
$$

To fulfill this relationship, the coefficients must satisfy the relations

$$
\begin{gathered}
d_{1}+d=7-2 \alpha, \\
d_{2}+d d_{1}+1=19-8 \alpha+4 c, \\
d_{3}+d d_{2}+d_{1}=26-12 \alpha+8 c-8 \alpha c, \\
1+d d_{3}+b_{2}=19-8 \alpha+4 c, \\
d+d_{3}=7-2 \alpha .
\end{gathered}
$$


From the first and the last relations, it follows that $d_{1}=d_{3}$. Clearing $d_{1}$ and $d_{2}$ in terms of $d$ in the first two equations, we get

$$
\begin{aligned}
& d_{1}=7-2 \alpha-d, \\
& d_{2}=18-8 \alpha+4 c-7 d+2 d \alpha+d^{2},
\end{aligned}
$$

and substituting in the third one, we obtain again the cubic equation:

$$
\begin{aligned}
& d^{3}+(-7+2 \alpha) d^{2}+(16-8 \alpha+4 c) d-12 \\
& \quad+8 \alpha-8 c-8 \alpha c=0
\end{aligned}
$$

For $(\alpha, c)$ real, the real solution of this polynomial is

$$
d(\alpha, c)=\frac{1}{3}(7-2 \alpha)-\frac{1}{3}(\sqrt[3]{f(\alpha, c)}+\sqrt[3]{g(\alpha, c)}),
$$

with $f$ and $g$ previously defined.

For $c=0$, then $f(\alpha, 0)=g(\alpha, 0)=(-1+2 \alpha)^{3}$; then,

$$
h_{1}(z, \alpha, 0)=1+b z+z^{2}=1+(3-2 \alpha) z+z^{2},
$$

whose zeros coincide with the strange fixed points of the Chebyshev-Halley family (see [1]).

Moreover, for $\alpha=0$, the cubic equation (51) is

$$
d^{3}-7 d^{2}+(16+4 c) d-8 c-12=0,
$$

whose solutions are

$$
\begin{gathered}
d=2, \quad d=\frac{5}{2}+\frac{1}{2} \sqrt{1-16 c}, \\
d=\frac{5}{2}-\frac{1}{2} \sqrt{1-16 c},
\end{gathered}
$$

and if $d=2$, then $d_{1}=5$ and $d_{2}=8+4 c$. So, the zeros of the function,

$$
h_{2}(z, 0, c)=1+5 z+8 z^{2}+4 z^{2} c+5 z^{3}+z^{4},
$$

coincide with the strange fixed points $z_{i}, i=1,2,3,4$, of the c-family (see [5]).

On the other hand, for $c=0$, the critical points are

$$
\begin{aligned}
& z_{1}(\alpha, 0)=\frac{3-4 \alpha+2 \alpha^{2}+\sqrt{\alpha(-2+\alpha)(-3+2 \alpha)(-1+2 \alpha)}}{3(-1+\alpha)}, \\
& z_{2}(\alpha, 0)=\frac{3-4 \alpha+2 \alpha^{2}-\sqrt{\alpha(-2+\alpha)(-3+2 \alpha)(-1+2 \alpha)}}{3(-1+\alpha)}, \\
& z_{3}(\alpha, 0)=z_{4}=z_{5}=z_{6}=-1
\end{aligned}
$$

which are the critical points of Chebyshev-Halley family (see [1]).

Finally, for $\alpha=0$, the critical points are

$$
\begin{aligned}
& z_{1}(0, c)=\frac{3+4 c+2 \sqrt{5 c(3-c)}}{3(-1+2 c)}, \\
& z_{2}(0, c)=\frac{3+4 c-2 \sqrt{5 c(3-c)}}{3(-1+2 c)}, \\
& z_{3}(0, c)=z_{4}=z_{5}=z_{6}=-1,
\end{aligned}
$$

which coincide with the critical points of $c$-family (see [5]).

\section{Study of the Parameter Space}

As we have said, the critical points whose analytical expression has been previously obtained satisfy that $z_{2 k-1}(\alpha, c)=$ $1 / z_{2 k}(\alpha, c)$, for $k=1,2,3$. So, only three of them are free critical points.

It is a known fact that any basin of attraction must hold, at least, one critical point (see, e.g., [10] or [11]). Then, the knowledge of the critical points and their behavior as a starting point of the iterative process is of vital importance. In this section, the parameter plane associated to each free critical point is obtained, by using them as starting points of the $(\alpha, c)$-family and taking note of their orbits depending on the real values of the parameters involved. So, in Figure 8 each point $(a, b)$ of the real plane $[-10,10] \times[-10,10]$ represents a scheme of the $(\alpha, c)$-family, for $\alpha=a$ and $c=b$. Then, the color of this point is drawn in red if this specific method converges to one of the roots $(0$ and $\infty$, after Möbius transformation), or in white in other case. Besides, the color will be brighter if the number of iterations needed to reach one of the roots is small and darker if it is high. The routines used to get these pictures are slightly modified from those appearing in [13]. Similar features can be found in [14].

So, we will observe the different parameter planes in order to find the stable behavior (in red) and the "dangerous" regions (in white). In the last ones, divergence, attracting strange fixed points or even attracting periodic orbits, can appear.

In Figure 8(a), the parameter plane associated to critical points $z_{1}$ and $z_{2}$ can be observed. Wide white areas of no convergence to the roots are found, but also the number of elements of the $(\alpha, c)$-family that can be used to converge to them is high.

A similar behavior can be found in Figures 8(b) and 8(c). Stable behavior appears in wide areas of the region where $\alpha>0$ and $c>0$ and also for $\alpha<0$ and $0<c<3$. In the following, we select different points in stable and unstable regions of the parameter planes (i.e., we choose some iterative methods of the class) and we see their specific dynamical behavior on quadratic polynomials.

\section{Dynamical Planes}

The analysis of dynamical planes of a parametric family or a method is a usual technique for improving the knowledge of a method; see, for example, [15-18] and references therein.

The generation of dynamical planes is very similar to the one of parameter spaces. In case of dynamical planes, the value of each parameter, $\alpha$ and $c$, is constant. So, each point of the complex plane is considered as a starting point of concrete element of the family of iterative methods and it is painted in different colors depending on the point which it has converged to. If it converges to 0 , then the point is painted in orange, when it converges to $\infty$, it appears in clear blue. If the iterates converge to a strange fixed point it is painted in different colors, as green or dark blue. Finally, the basins of attraction of periodic orbits are painted in black. As in the case of the parameter planes, routines appearing in [13] have been used. 


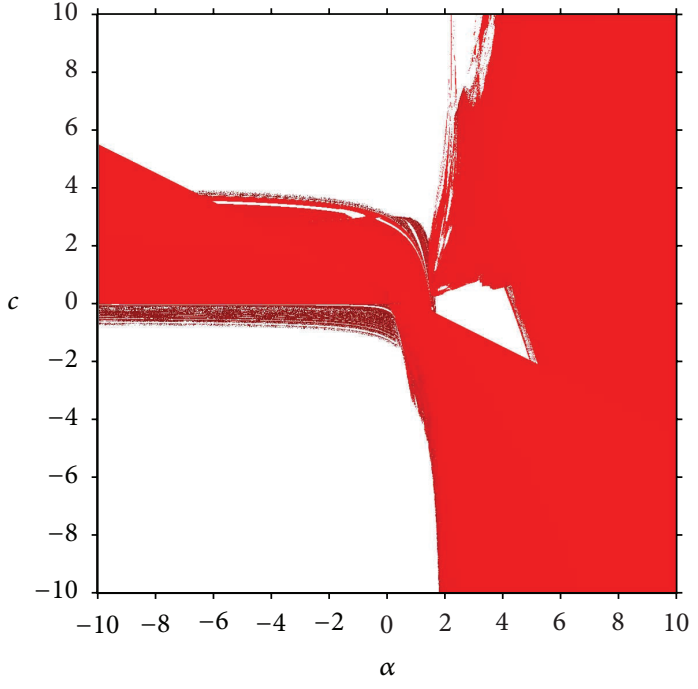

(a) Critical points $z_{1}$ and $z_{2}$

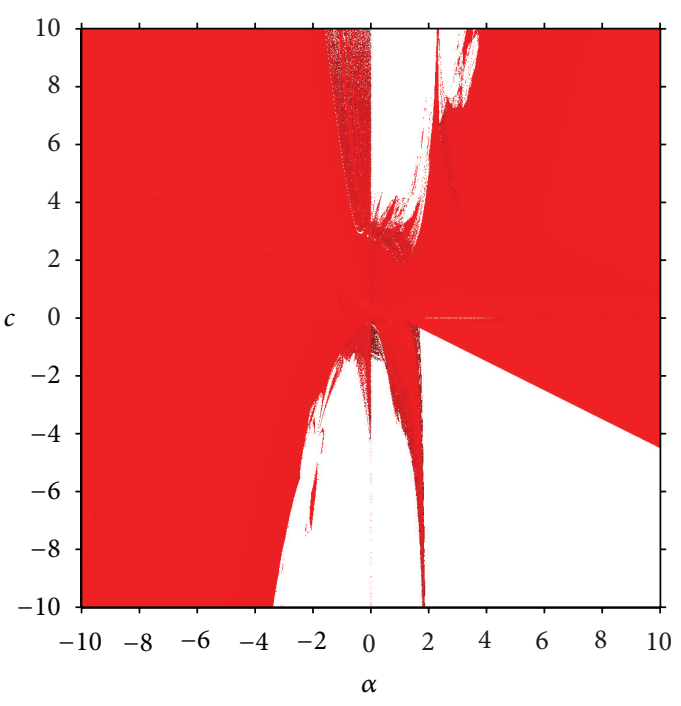

(b) Critical points $z_{3}$ and $z_{4}$

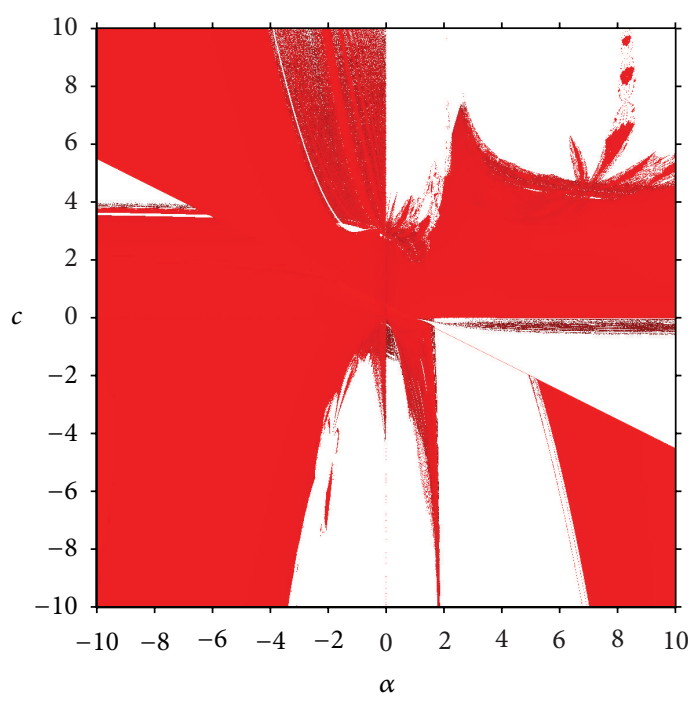

(c) Critical points $z_{5}$ and $z_{6}$

Figure 8: Parameter planes corresponding to different critical points.

Firstly, let us take into account the information obtained in the parameter planes (Figure 8). The most stable behavior corresponds to those $(\alpha, c)$ values that remain in red at every parameter plane: they correspond to methods of the family that, using any free critical point as an initial estimation, it only converges to zero or infinite, that is, to any of the roots of the original quadratic polynomial. However, it can be seen at Figure 9 that all these dynamical planes are not equally useful, as the wideness of the immediate basin of attraction is clearly bigger in some cases.

With respect to the unstable behavior, in Figures 10 to 12, different situations can be observed. Specifically, in Figures 10(a) and 10(b), two periodic orbits of period 4 are found for values of the parameters $\alpha=5$ and $c=5$. Indeed, in Figure 11(b), a two-periodic orbit is observed for $\alpha=c=10$, with a wide basin of attraction in black. In Figure 11(a), a twoperiodic orbit is found, but also an attracting strange fixed point whose basin of attraction is much bigger than the one of $z=0$. Finally, in Figure 12, some dynamical planes with four different basins of attraction are shown, two of them corresponding to attracting strange fixed points.

Let us remark that in all cases, a cubic symmetry is observed. This fact is due to the symmetry of the polynomials (with respect to their coefficients) involved in the rational operator associated to the family.

\section{Conclusions}

In this paper, the dynamical behavior of the $(\alpha, c)$-family has been studied, confirming that it is much more than the union of cases $\alpha=0$ ( $c$-family) and $c=0$ (Chebyshev-Halley family), as there exist hybrid iterative methods with specially stable behavior. The fixed and critical points of the rational 


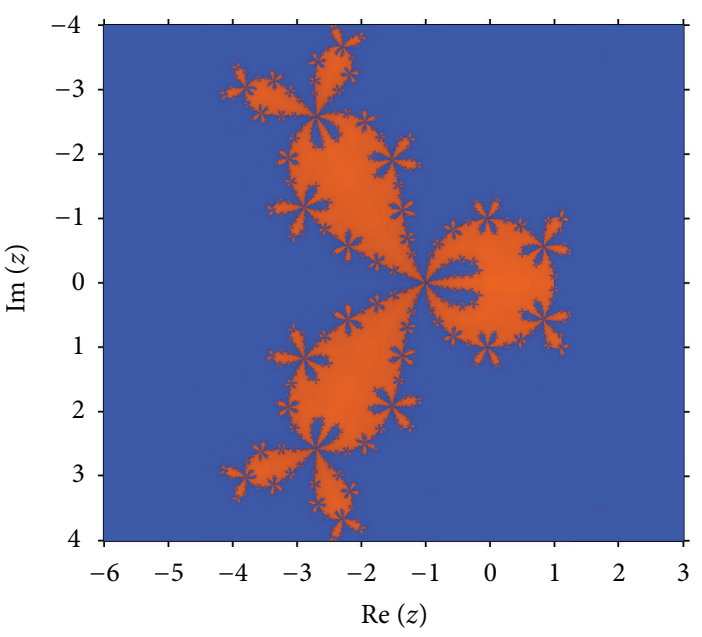

(a) $\alpha=9, c=3$

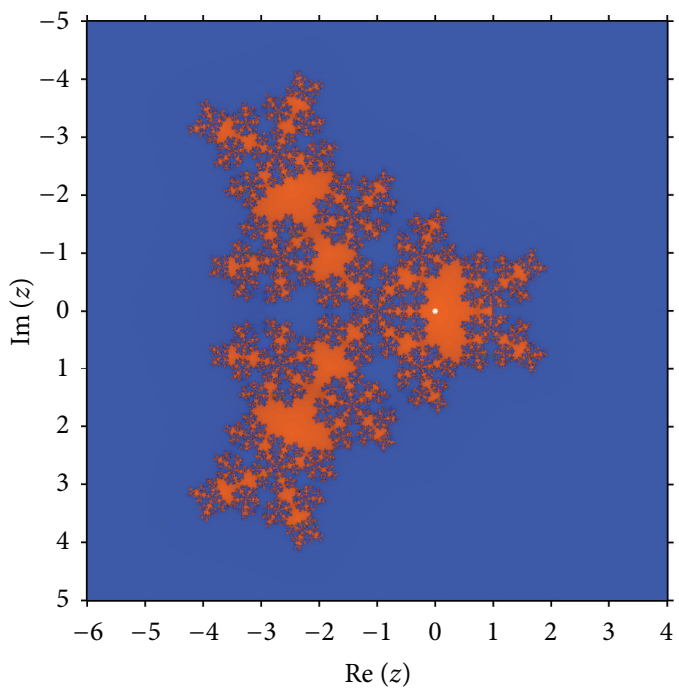

(c) $\alpha=-10, c=7 / 2$

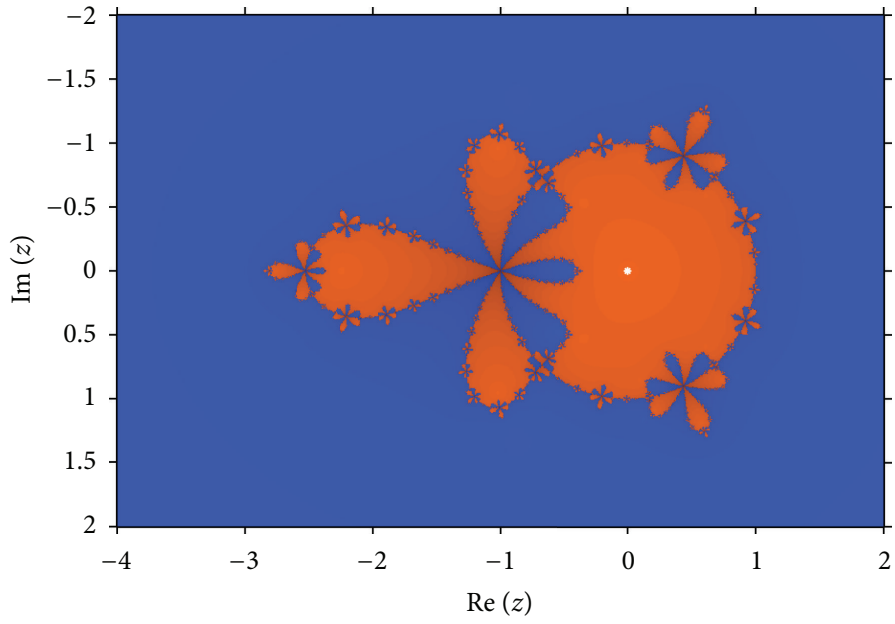

(b) $\alpha=1 / 2, c=-1 / 5$

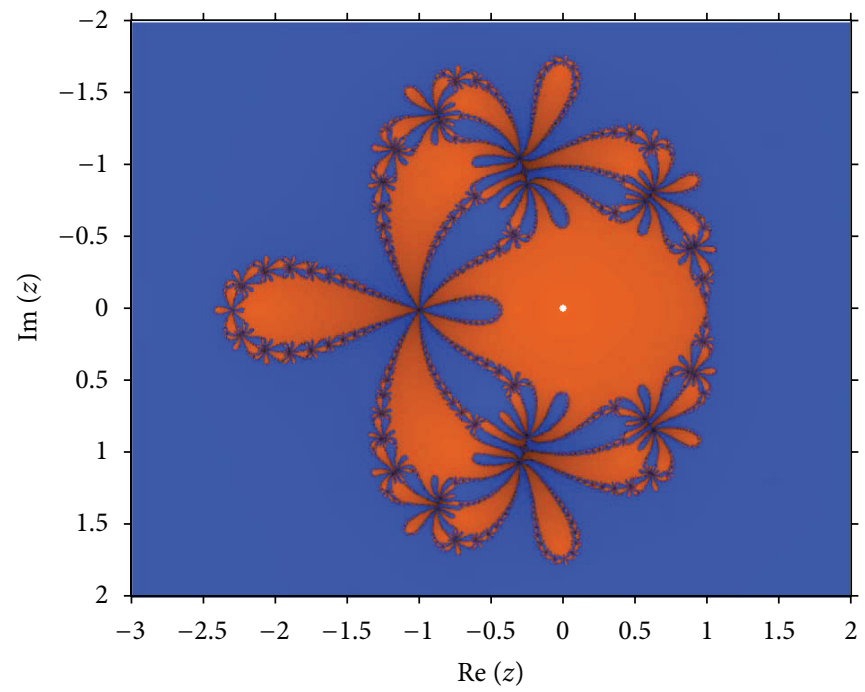

(d) $\alpha=-5, c=-1 / 5$

FIgure 9: Stable behavior.

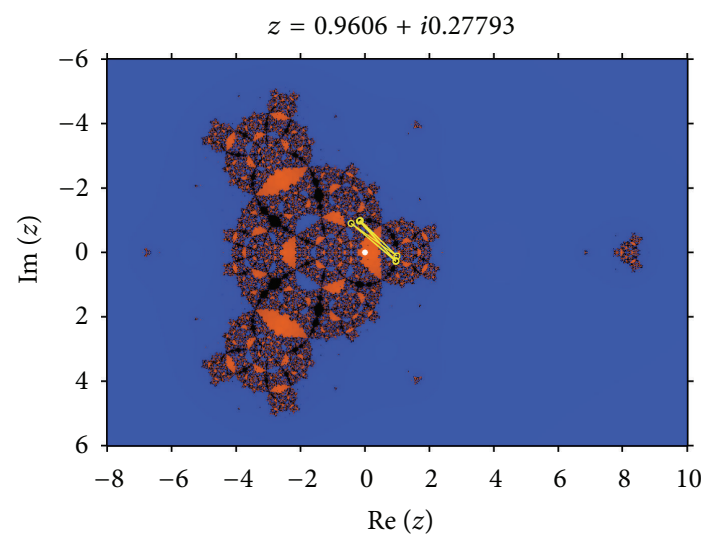

(a) $\alpha=5, c=5$

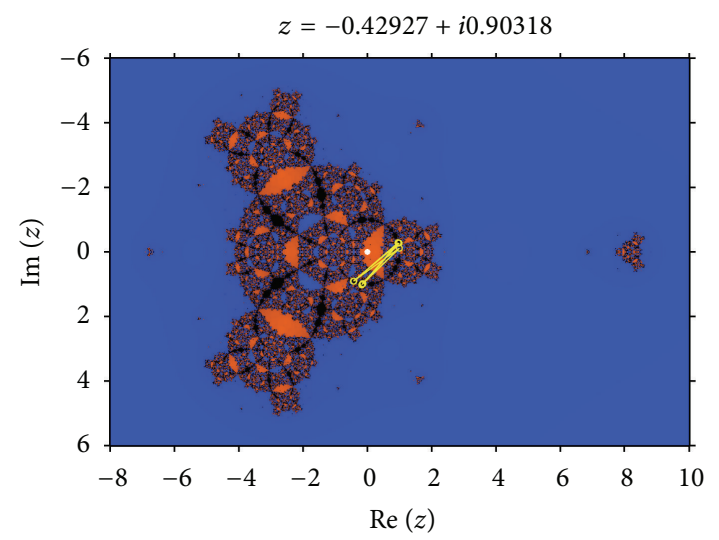

(b) $\alpha=5, c=5$

FIGURE 10: Unstable behavior: two 4-periodic orbits. 


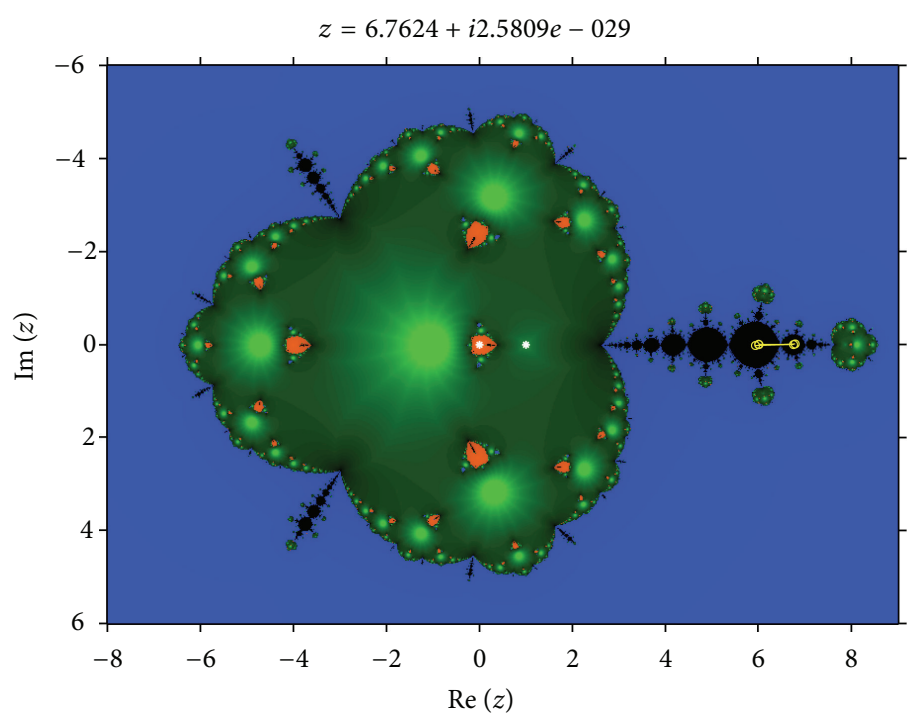

(a) $\alpha=5, c=-5$

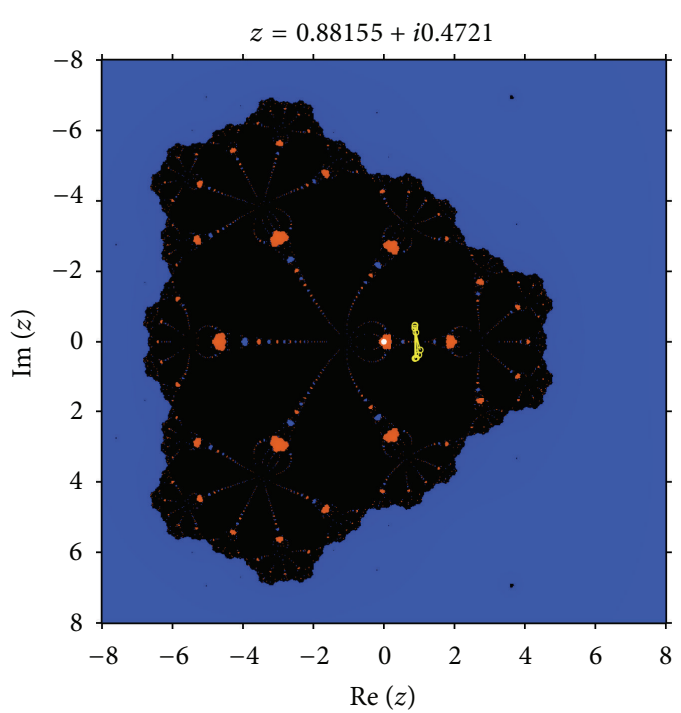

(b) $\alpha=10, c=10$

FIGURE 11: Unstable behavior: one 2-periodic orbit.

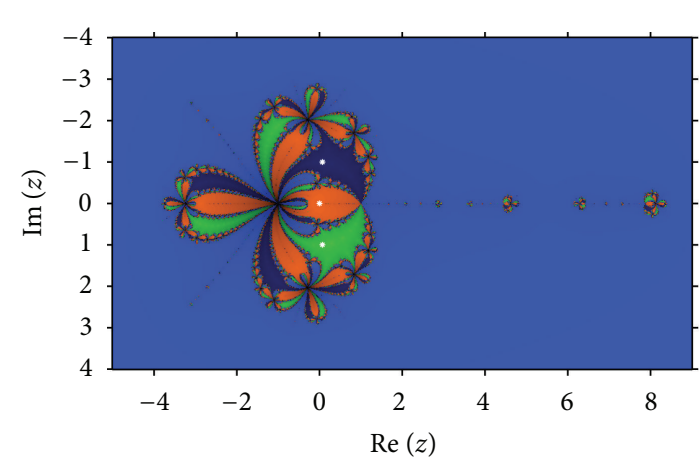

(a) $\alpha=5, c=-1$

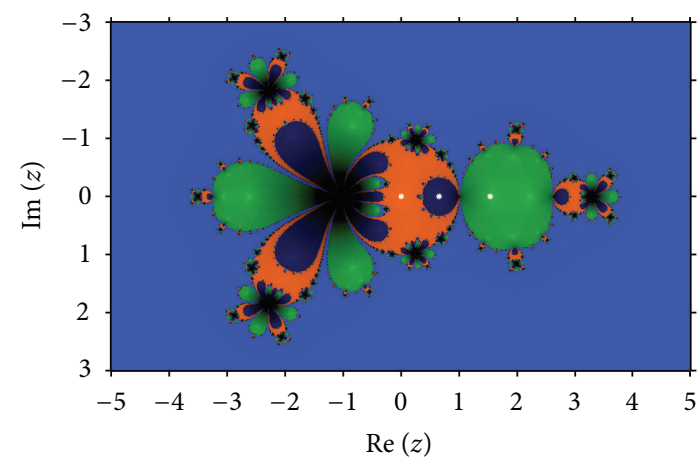

(b) $\alpha=2.5, c=-1$

FIGURE 12: Unstable behavior: attracting strange fixed points.

operator associated to the family on quadratic polynomials were obtained, and the regions in which these points are real or complex have been shown. From critical points, the parameter planes of the family were drawn and some regions of very stable and unstable behavior have been detected. Dynamical planes for specific values of the parameters in these regions were shown.

\section{Conflict of Interests}

The authors declare that there are no conflict of interest regarding the publication of this paper.

\section{Acknowledgment}

This work was supported by Ministerio de Ciencia y Tecnología MTM2011-28636-C02-\{01,02\}, P11B2011-30 of the Universitat Jaume I, and PAID-SP20120474 of the Universitat Politècnica de València.

\section{References}

[1] A. Cordero, J. R. Torregrosa, and P. Vindel, "Dynamics of a family of Chebyshev-Halley type methods," Applied Mathematics and Computation, vol. 219, no. 16, pp. 8568-8583, 2013.

[2] A. Cordero, J. R. Torregrosa, and P. Vindel, "Period-doubling bifurcations in the family of Chebyshev-Halley-type methods," International Journal of Computer Mathematics, vol. 90, no. 10, pp. 2061-2071, 2013.

[3] A. Cordero, J. R. Torregrosa, and P. Vindel, "Bulbs of period two in the family of Chebyshev-Halley iterative methods on quadratic polynomials," Abstract and Applied Analysis, vol. 2013, Article ID 536910, 10 pages, 2013.

[4] A. Cordero, J. García-Maimó, J.R. Torregrosa, M. P. Vassileva, and P. Vindel, "Chaos in King's iterative family," Applied Mathematics Letters, vol. 26, no. 8, pp. 842-848, 2013.

[5] B. Campos, A. Cordero, J. R. Torregrosa, and P. Vindel, "Dynamics of the family of c-iterative methods," International Journal of Computer Mathematics, 2014. 
[6] J. M. Gutiérrez and M. A. Hernández, "A family of ChebyshevHalley type methods in Banach spaces," Bulletin of the Australian Mathematical Society, vol. 55, no. 1, pp. 113-130, 1997.

[7] M. A. Hernandez and M. A. Salanova, "A family of ChebyshevHalley type methods," International Journal of Computer Mathematics, vol. 47, no. 1-2, pp. 59-63, 1993.

[8] S. Amat, S. Busquier, and S. Plaza, "On the dynamics of a family of third-order iterative functions," The ANZIAM Journal, vol. 48, no. 3, pp. 343-359, 2007.

[9] S. Amat, C. Bermudez, S. Busquier, and S. Plaza, "Review of some iterative root-finding methods from a dynamical point of view," Scientia A: Mathematical Sciences, vol. 10, pp. 3-35, 2004.

[10] P. Blanchard, "Complex analytic dynamics on the Riemann sphere," Bulletin: New Series of the American Mathematical Society, vol. 11, no. 1, pp. 85-141, 1984.

[11] A. F. Beardon, Iteration of Rational Functions, vol. 132 of Graduate Texts in Mathematics, Springer, New York, NY. USA, 1991.

[12] B. Campos, A. Cordero, A. Magre, J. R. Torregrosa, and P. Vindel, "Bifurcations of the roots of a 6-degree symmetric polynomial coming from the fixed point operator of a class of iterative methods," in Proceedings of CMMSE, 2014.

[13] F. Chicharro, A. Cordero, and J. R. Torregrosa, "Drawing dynamical and parameter planes of iterative families and methods," The Scientific World Journal, vol. 2013, Article ID 780153, 11 pages, 2013.

[14] A. A. Magreñan, "A new tool to study real dynamics: the convergence plane," http://arxiv.org/abs/1310.3986.

[15] F. Chicharro, A. Cordero, J. M. Gutiérrez, and J. R. Torregrosa, "Complex dynamics of derivative-free methods for nonlinear equations," Applied Mathematics and Computation, vol. 219, no. 12, pp. 7023-7035, 2013.

[16] C. Chun, M. Y. Lee, B. Neta, and J. Dzunic, "On optimal fourthorder iterative methods free from second derivative and their dynamics," Applied Mathematics and Computation, vol. 218, no. 11, pp. 6427-6438, 2012.

[17] B. Neta, M. Scott, and C. Chun, "Basin attractors for various methods for multiple roots," Applied Mathematics and Computation, vol. 218, no. 9, pp. 5043-5066, 2012.

[18] M. Scott, B. Neta, and C. Chun, "Basin attractors for various methods," Applied Mathematics and Computation, vol. 218, no. 6, pp. 2584-2599, 2011. 


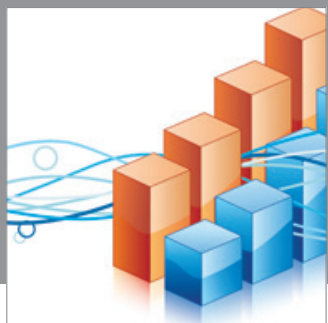

Advances in

Operations Research

mansans

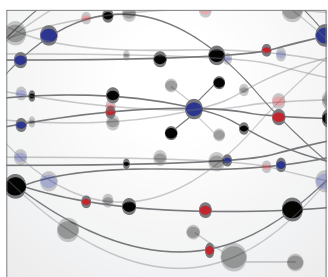

The Scientific World Journal
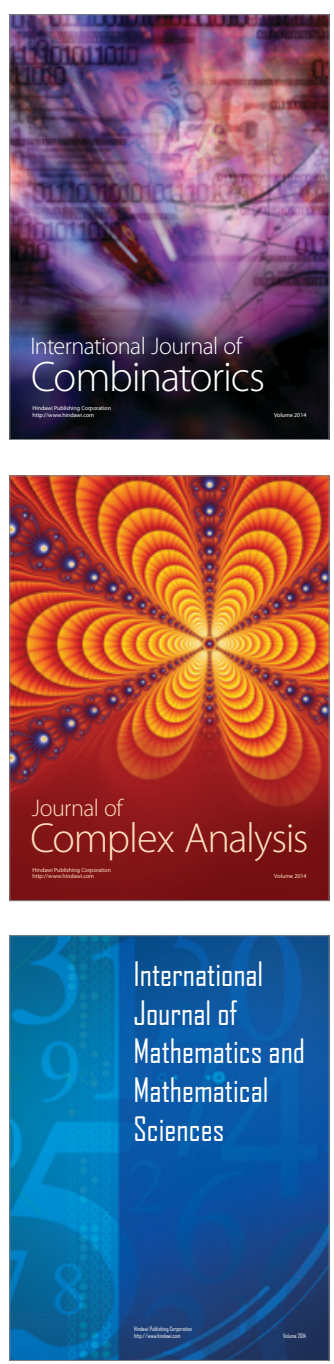
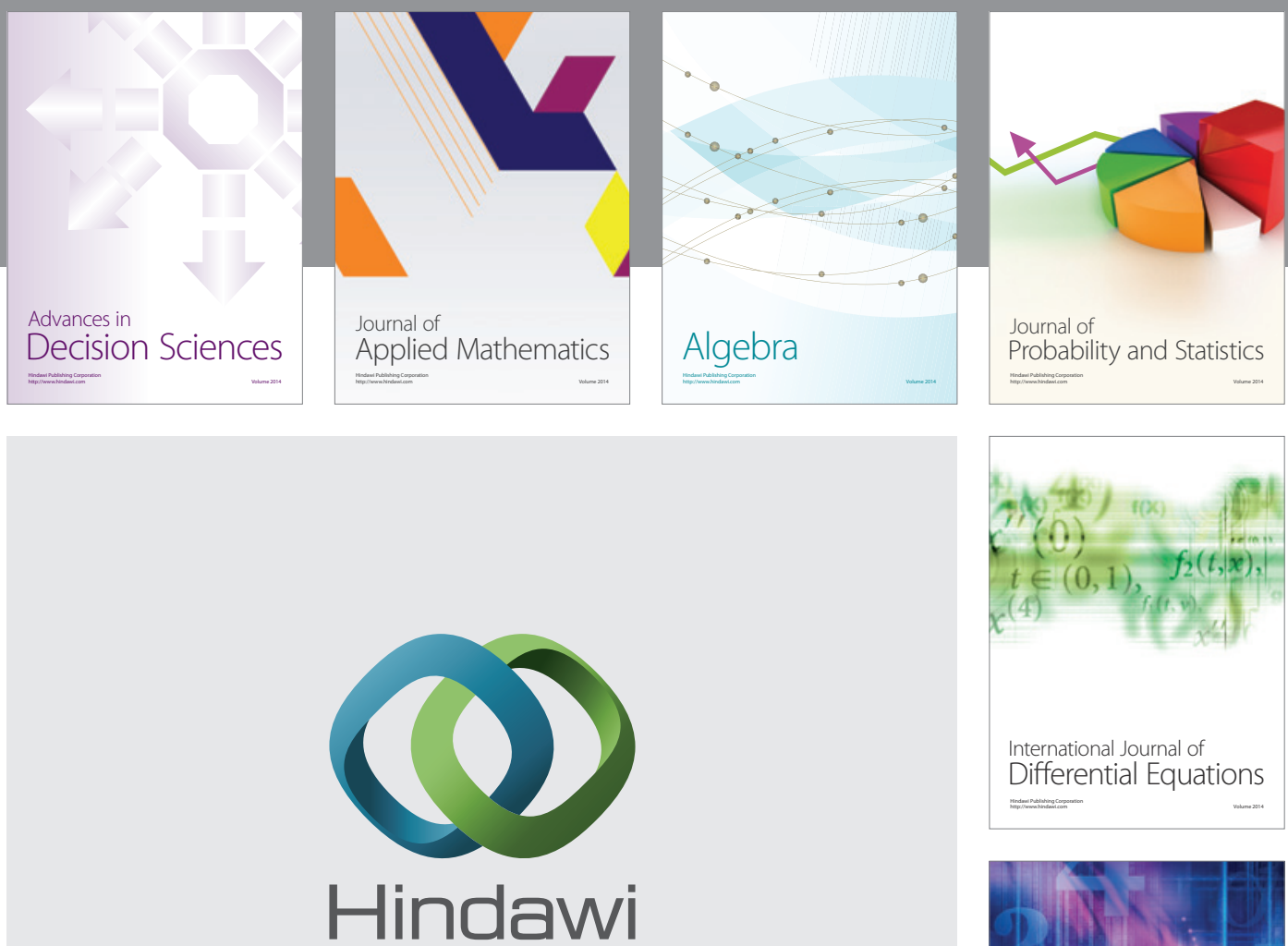

Submit your manuscripts at http://www.hindawi.com
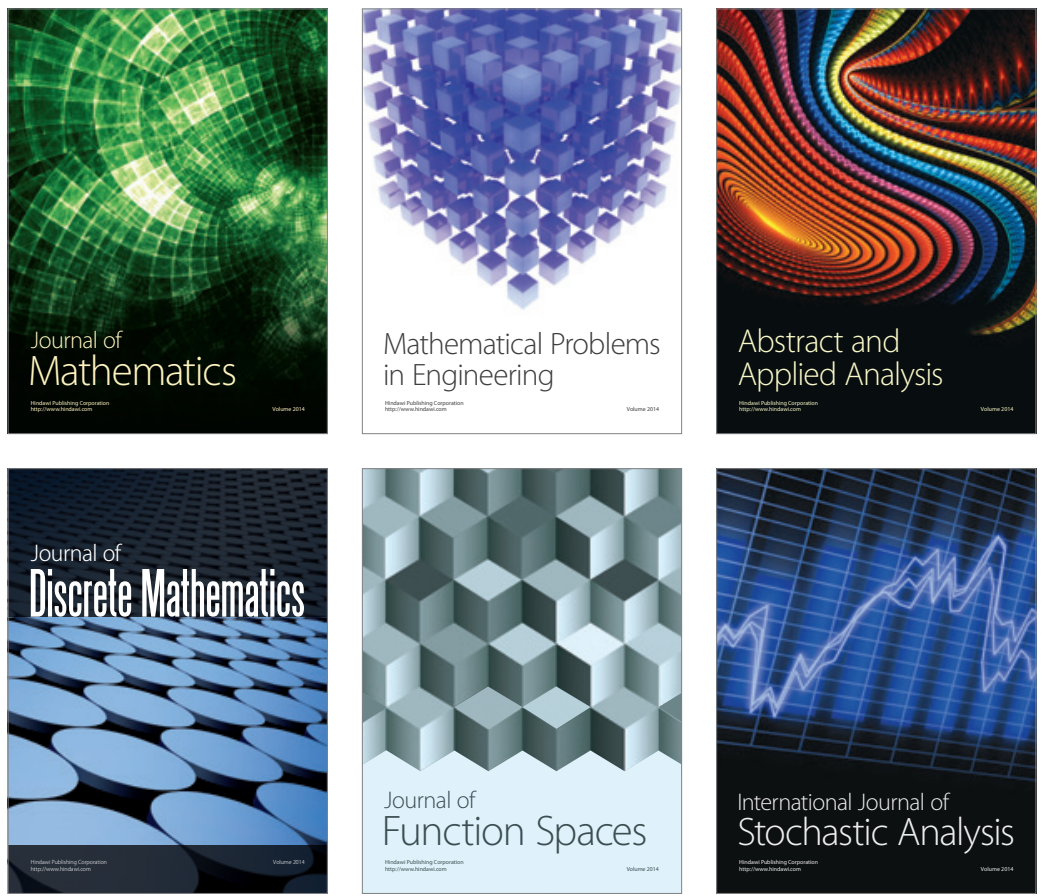

Journal of

Function Spaces

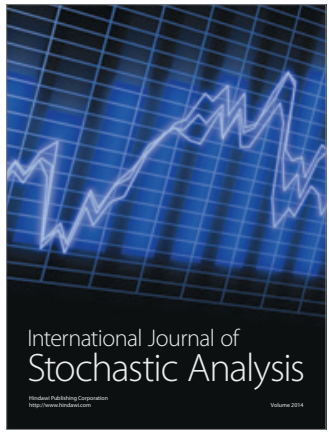

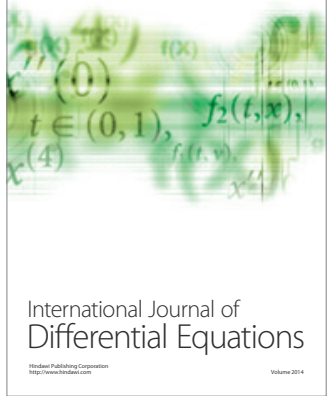
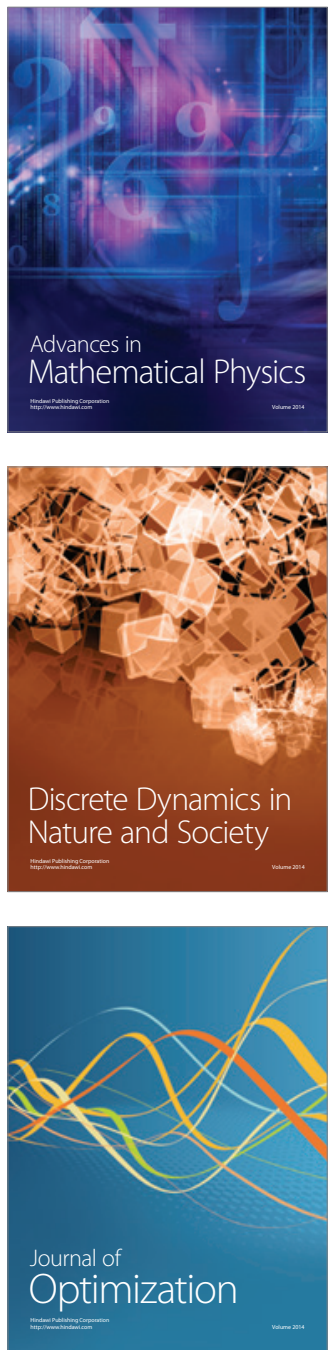